\title{
Magnetic fields of massive stars
}

\author{
Swetlana Hubrig ${ }^{1}$, Michel Curé ${ }^{2}$, Ilya Ilyin ${ }^{1}$, and Markus Schöller ${ }^{3}$ \\ ${ }^{1}$ Astrophysikalisches Institut Potsdam, An der Sternwarte 16, Potsdam, Germany \\ email: shubrig@aip.de \\ ${ }^{2}$ Departamento de Fisica y Astronomía, Facultad de Ciencias, \\ Universidad de Valparaíso, Chile \\ ${ }^{3}$ European Southern Observatory, Karl-Schwarzschild-Str. 2, \\ 85748 Garching bei München, Germany
}

\begin{abstract}
We recently carried out a spectropolarimetric study of a sample of massive O-type stars and pulsating $\beta$ Cephei stars using the SOFIN echelle spectrograph at the $2.56 \mathrm{~m}$ Nordic Optical Telescope and the low-resolution FORS 2 spectrograph at the VLT in spectropolarimetric mode. The sample consists of massive stars already detected as magnetic in the course of our previous low-resolution polarimetric observations with FORS 1 and a few O-type stars with magnetic field detections reported in the literature.
\end{abstract}

Keywords. stars: magnetic fields, techniques: polarimetric, stars: early-type, stars: rotation, stars: oscillations, stars: kinematics, stars: individual (HD 108, HD 191612, V1449 Aql, $\xi^{1}$ CMa)

\section{Observations and analysis}

To date, only a small number of O, early B-type, and Wolf-Rayet stars have been investigated for magnetic fields, and as a result, only about two dozen magnetic massive early type stars are known. On the other hand, a lot of effort has been put into the research of massive stars in recent years in order to properly model the effects of rotation, stellar winds, surface chemical composition, and evolution. Polarimetric spectra of the massive O-type stars HD 108, HD 36879, 15 Mon, 9 Sgr, and HD 191612 were obtained with the SOFIN spectrograph, which is equipped with three optical cameras and mounted at the Cassegrain focus of the NOT. The new magnetic field measurements confirm the presence of longitudinal magnetic fields in these stars (e.g. Hubrig et al. 2008, 2009a). The presence of a longitudinal magnetic field in the Of?p star HD 108, $\left\langle B_{z}\right\rangle=-150 \pm 10 \mathrm{G}$, was recently reported by Martins et al. (2010), using NARVAL and ESPaDOnS observations. For this star, Nazé et al. (2006) suggested a period between 50 and 60 years. Applying the moment technique, we obtain a mean longitudinal magnetic field $\left\langle B_{z}\right\rangle=-168 \pm 35 \mathrm{G}$. A mean longitudinal magnetic field $\left\langle B_{z}\right\rangle=-220 \pm 38 \mathrm{G}$ in the Of?p star HD 191612 was measured by Donati et al. (2006), combining spectropolarimetric observations from four nights. The rotation period of $537.6 \mathrm{~d}$ was determined by Howarth et al. (2007). Using ephemeris from this work, the magnetic field measurements of Donati et al. (2006) have been carried out at a rotation phase of $\sim 0.24$. Our measurements using eight spectral lines result in $\left\langle B_{z}\right\rangle=450 \pm 153 \mathrm{G}$ at rotation phase 0.43 . The difference in phase between the measurement of Donati et al. (2006) and our measurement is about 0.19 . Thus, we observe a change of polarity over $\sim 100$ days.

Over several years, we undertook a magnetic field survey for main-sequence pulsating B-type stars, namely the slowly pulsating B (SPB) stars and $\beta$ Cephei stars, with FORS $1 / 2$ in spectropolarimetric mode at the VLT, allowing us to detect in four $\beta$ Cephei stars and 16 SPB stars, for the first time, longitudinal magnetic fields of the order of a few hundred Gauss. The SOFIN measurements of $\xi^{1} \mathrm{CMa}$, separated by one year, 


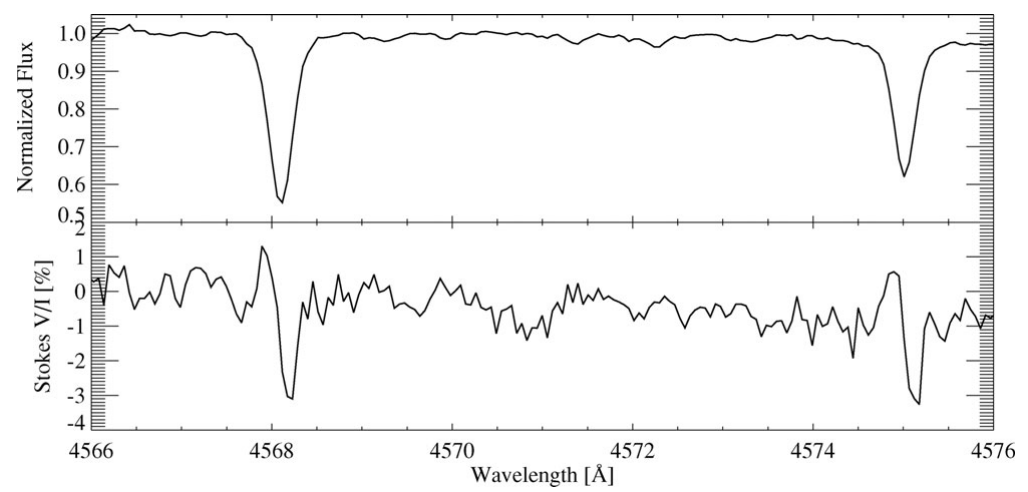

Figure 1. SOFIN $I$ and $V$ spectra of $\xi^{1} \mathrm{CMa}$ in the spectral region around the Si III (Mult. 2) lines.

$\left\langle B_{z}\right\rangle=386 \pm 139 \mathrm{G}$ and $\left\langle B_{z}\right\rangle=297 \pm 126 \mathrm{G}$, confirmed our previous detection of a slightly variable magnetic field in this star (Hubrig et al. 2006). In Fig. 1 we present SOFIN $I$ and $V$ spectra of this star in the spectral region around the Si III (Mult. 2) lines. Another $\beta$ Cephei star, V1449 Aql, previously reported by Hubrig et al. (2009b) as magnetic, was found to show the strongest mean longitudinal magnetic field among the magnetic $\beta$ Cephei stars, of the order of $-800 \mathrm{G}$. The detection of such a strong magnetic field in this massive star is of special interest due to the recently discovered solar-like pulsations using CoRoT observations (Belkacem et al. 2009).

\section{Summary}

Although it was possible to recognize a few hot magnetic stars as being peculiar on the basis of their spectral morphology prior to their field detection (Walborn 2006), the presence of a magnetic field can also be expected in stars of other classification categories. Our measurements of several massive stars indicate that magnetic fields are possibly present in stars with very different observed properties in visual, X-ray, and radio domains. Future magnetic field measurements of massive stars in field and cluster stars will constrain the conditions controlling the presence of magnetic fields, and the implications of these fields on their mass-loss rate and evolution.

\section{References}

Belkacem, K., Samadi, R., Goupil, M.-J., Lefèvre, L. et al. 2009, Science 324, 1540

Donati, J.-F., Howarth, I. D., Bouret, J.-C., Petit, P. et al. 2006, MNRAS 365, L6

Howarth, I. D., Walborn, N. R., Lennon, D. J., Puls, J. et al. 2007, MNRAS 381, 433

Hubrig, S., Briquet, M., Schöller, M., De Cat, P. et al. 2006, MNRAS 369, L61

Hubrig, S., Schöller, M., Schnerr, R. S., González, J. F. et al. 2008, A\&̛A 490, 793

Hubrig, S., Stelzer, B., Schöller, M., Grady, C. et al. 2009a, A\&SA 502, 283

Hubrig, S., Briquet, M., De Cat, P., Schöller, M. et al. 2009b, AN 330, 317

Martins, F., Donati, J.-F., Marcolino, W. L. F., Bouret, J.-C. et al. 2010, MNRAS 407, 1423

Nazé, Y., Barbieri, C., Segafredo, A., Rauw, G. et al. 2006, IBVS 5693

Walborn, N. R. 2006, in: The Ultraviolet Universe: Stars from Birth to Death, Proc. IAU Joint Discussion 4, \#19, Prague, Czech Republic 